\title{
Mise en scène de la «bonne entente » interreligieuse et reconnaissance
}

Anne-Sophie Lamine

\section{(2) OpenEdition}

1 Journals

Édition électronique

URL : http://journals.openedition.org/assr/1112

DOI : $10.4000 /$ assr. 1112

ISSN : $1777-5825$

Éditeur

Éditions de l'EHESS

\section{Édition imprimée}

Date de publication : 1 janvier 2005

Pagination : 83-96

ISBN : 2-7132-2043-2

ISSN : 0335-5985

\section{Référence électronique}

Anne-Sophie Lamine, « Mise en scène de la «bonne entente » interreligieuse et reconnaissance », Archives de sciences sociales des religions [En ligne], 129 | janvier - mars 2005, mis en ligne le 09 janvier 2008, consulté le 19 avril 2019. URL : http://journals.openedition.org/assr/1112 ; DOI : 10.4000/ assr.1112

Ce document a été généré automatiquement le 19 avril 2019

() Archives de sciences sociales des religions 


\title{
Mise en scène de la « bonne entente » interreligieuse et reconnaissance
}

\author{
Anne-Sophie Lamine
}

1 Les religions prennent aujourd'hui de plus en plus ouvertement place dans l'espace public, en particulier par le rôle social que jouent diverses associations confessionnelles ${ }^{1}$. De plus, face à la diversification du champ religieux, les villes élaborent des régulations locales $^{2}$. L'observation des relations interreligieuses, et plus largement la mise en scène d'une pluralité religieuse coopérative, est aussi révélatrice des transformations induites par la diversité religieuse. La pluralité est tout d'abord prise en compte par les groupes religieux. Elle a ensuite des effets sur les identités religieuses individuelles et collectives. Elle a aussi favorisé le développement d'un mouvement interreligieux associatif. Enfin, elle induit des mutations des rapports entre les représentants de l'État et les groupes religieux ${ }^{3}$. Cet article se propose d'analyser ce dernier type de transformation, dans sa dimension plurireligieuse ${ }^{4}$, qui touche directement la mise en œuvre du principe de laïcité. Ces changements sont particulièrement visibles dans deux aspects du rapport entre les religions et la sphère publique. D'une part, on constate que les pouvoirs publics et leurs représentants se mettent à promouvoir la «bonne entente » entre les religions. D'autre part, il apparaît que l'État commence à prendre en compte l'écart entre l'égalité formelle et l'égalité réelle en matière religieuse et à reconnaître les religions « minoritaires ${ }^{5}$ en les mettant, au moins symboliquement, en situation d'égalité avec la religion majoritaire.

Quand les pouvoirs publics promeuvent la collaboration interreligieuse

2 Les pouvoirs publics font face à un accroissement des demandes liées à la pluralisation religieuse de la population : lieux de culte, carrés dans les cimetières, prise en compte de besoins particuliers dans les hôpitaux, prisons ou armées. Au-delà des réponses diversifiées apportées à ces requêtes, on observe aussi une multiplication d'initiatives publiques visant à promouvoir la «bonne entente des religions". Si certaines villes sont initiatrices de conseils interreligieux ou de projets favorisant la connaissance du fait 
religieux, bien plus nombreuses sont les institutions publiques ou les responsables politiques qui soutiennent ou initient des manifestations plurireligieuses ponctuelles, en particulier en temps de crise.

Des villes initiatrices de projets plurireligieux

3 Trois municipalités ont développé des projets plurireligieux assez élaborés : Marseille, Roubaix et Montreuil ${ }^{6}$. Même si ces villes ont poussé plus loin que d'autres la logique de promotion de la pluralité religieuse coopérative, ces expériences sont significatives de la mutation des rapports entre autorités locales et groupes religieux.

Marseille-Espérance est une structure "inter-communautaire ", fondée en 1990 par le maire (R. Vigouroux, socialiste) d'une ville qui compte d'importantes minorités religieuses ${ }^{7}$. Dans un contexte marqué par la montée du Front National et par des débats houleux sur un projet de mosquée, la crise du Golfe fait craindre des conflits ethnicoreligieux. Présidée par le maire (ou son adjoint), rattachée au cabinet du maire puis à la direction de la communication, la structure est un partenariat à la fois informel et officiel entre les religions et la mairie, orienté vers le "vivre ensemble». Ses membres sont les sept personnalités religieuses (de rang le plus élevé pour la région) et leur sept délégués ${ }^{8}$, le maire, son adjoint, un coordinateur (prêtre catholique), aidés d'une chargée de mission de la mairie. Lors de chaque crise susceptible d'avoir des répercussions locales, MarseilleEspérance fait une déclaration largement médiatisée ${ }^{9}$, dans laquelle elle n'hésite pas à marquer sa dimension religieuse. Ainsi, le 14 septembre 2001, elle condamne «le fanatisme religieux, d'où qu'il vienne » et en conclut par: " nous prions le Dieu auquel nous croyons pour qu'Il fasse de nous des semeurs de concorde et de paix ». En 2002, les membres musulmans, chrétiens et bouddhistes accompagnent l'enterrement des rouleaux de la Torah, suite à l'incendie d'une synagogue. Les membres participent aux événements des autres communautés. Un calendrier mentionnant les fêtes des sept religions est tiré à 15000 exemplaires. Le gala inter-communautaire annuel accueille quelque 5000 spectateurs et les colloques grand public 400 à 800 participants. La structure parraine des évènements comme des tournois sportifs ou encore un lieu interconfessionnel de prières dans un hôpital ${ }^{10}$. En 2000, lors du XXVI centenaire de la cité, seize "places de l'Espérance» sont inaugurées ainsi que l'Arbre de l'Espérance, sculpture métallique monumentale, au pied de laquelle sont gravés les noms de 300000 Marseillais qui se sont associés à la démarche. Ces actions sont soutenues par un important travail de communication : on montre «que les chefs religieux sont amis » et que «les juifs et les musulmans peuvent s'entendre ». Le groupe reçoit de nombreuses demandes d'interventions dans des établissements éducatifs ou des structures associatives et institutionnelles, françaises et étrangères. L'absence de statut permet la souplesse de fonctionnement et le soutien de la mairie confère la légitimité et les moyens. Un membre souligne le risque d'instrumentalisation, en observant que pour le maire: "c'est bon à la fois politiquement et électoralement». Du côté des religieux, chacun assure poursuivre cette pédagogie en "interne» et chaque communauté retire des avantages de ce travail commun, en termes de reconnaissance et d'image d'ouverture.

5 Très multiculturelle aussi, Roubaix est une ville ouvrière qui a vu une forte avancée de l'extrême droite en 1995, puis la parution d'un livre la décrivant comme majoritairement musulmane et gagnée par un islam radical visant à y appliquer la charia ${ }^{11}$. La mairie envisage dès 1996 de soutenir des initiatives interreligieuses en concertation avec les « humanistes ». En 1998, à la demande du maire, la Fédération des Associations Laïques (FAL) met en place Roubaix-Espérance, une association loi de 1901. Les délégués sont 
catholique (vicaire épiscopal), musulmans (six associations ${ }^{12}$ ), bouddhistes (trois pagodes), protestants (réformés et baptistes) ${ }^{13}$ et agnostiques, membres du cercle Condorcet et de la FAL, dont le président est aussi celui de Roubaix-Espérance jusqu'en 2002 (le président de la pagode cambodgienne lui succède). En plus de la participation aux évènements religieux, l'association publie une revue de presse sur les faits religieux, organise des conférences sur la laïcité ou sur les rapports entre religion et culture et une fête annuelle de l'amitié. Lors des journées du patrimoine, en collaboration avec l'office du tourisme, elle propose un circuit de visite des lieux de culte qui accueillent 400 à 700 visiteurs. En 2001, elle est sollicitée par la mairie pour proposer une liste de fêtes que les cantines scolaires doivent marquer d'un menu particulier ${ }^{14}$. Lorsqu'en 2002, la mairie définit un "schéma directeur d'intervention sur les lieux de culte ", elle mentionne les possibilités d'action à l'égard des associations cultuelles ${ }^{15}$, rappelle que les églises catholiques (antérieures à 1905) relèvent du patrimoine public, s'engage à examiner les « demandes de création, réparation et extension de lieux de cultes » et liste les opérations prévues visant des lieux de culte musulmans et bouddhistes et le temple protestant réformé. Si les objectifs de Roubaix-Espérance concernent le vivre ensemble et la promotion de la laïcité, la mairie se préoccupe aussi fortement de la régulation des associations musulmanes, de la transparence de leurs financements et de la mise en conformité de leurs lieux de culte.

6 À Montreuil (Seine-Saint-Denis), parallèlement à des démarches visant à faciliter l'implantation d'une synagogue et d'une mosquée et la création de carrés juifs et musulmans au cimetière, en 2002, la mairie met en place un Centre Civique d'Études du Fait Religieux (CCEFR) ${ }^{16}$. Des cycles de cours, assurés par des universitaires et destinés en priorité aux acteurs sociaux, éducatifs et associatifs, sont suivis par 30 à 40 personnes ${ }^{17}$. Des conférences touchent 150 à 400 personnes. Les programmes sont élaborés par un Conseil scientifique d'universitaires, présidé par Mohammed Arkoun. En s'appuyant sur ce professeur de la Sorbonne, invité dans plusieurs universités américaines, assez critique de l'islam populaire, le maire souhaite favoriser une certaine version du religieux, qui valorise la dimension culturelle, la faible visibilité ou l'invisibilité des signes religieux et une approche laïque ou théologiquement libérale de la pensée religieuse. La promotion de la connaissance des religions apparaît donc bien comme une sorte d'antidote laïque à la discrimination comme au "communautarisme ${ }^{18}$. La mairie convoque aussi plusieurs réunions interreligieuses par an, avec une quarantaine de représentants catholiques, protestants, juifs et musulmans, afin d'informer de ses projets et de promouvoir la laïcité, qui a été le thème de deux de ces rencontres. Lors de la signature du bail emphytéotique pour la synagogue, puis pour la mosquée, elle organise également une réception en présence des représentants des autres religions ${ }^{19}$. Cette démarche est un exemple typique de mise en scène républicaine du vivre ensemble et d'une régulation municipale d'un religieux citoyen et «laïquement compatible». D'autres mairies envisagent de mettre en place de telles structures ou ont déjà commencé à le faire. Le Groupe "Concorde et Solidarité », initié par la mairie de Lyon en 2002, "réunit l'ensemble des représentants des grandes religions [...] avec le maire de Lyon» dans un «engagement solidaire en faveur de la concorde civile, du dialogue et de la cohésion sociale ${ }^{20}$. On peut encore mentionner des manifestations plus culturelles et festives ayant une dimension interethnique et même interreligieuse, comme les soirées musicales de ramadan, organisées annuellement à l'Hôtel de Ville de Paris (depuis 2001), où le maire souhaite « rassembler [...] les parisiens de toutes les cultures et influences religieuses ${ }^{21}$. 
Une nouvelle pratique politique : susciter des déclarations de concorde interreligieuse

7 Les initiatives interreligieuses ponctuelles se sont multipliées surtout en temps de crise, depuis la guerre du Golfe (1991). Cette date est un tournant dans la prise en compte sociale de la pluralité religieuse, par crainte des répercussions possibles du conflit en France. Alors que certains responsables religieux font des déclarations communes au plan national et local, une nouvelle pratique politique se développe : des maires ou des préfets invitent des responsables religieux à faire des déclarations de paix. Ces initiatives se renouvellent au début de la seconde Intifada, lors de la multiplication des actes antisémites en France et lors de la guerre d'Irak. On peut en citer quelques-unes à titre d'exemple. À Lille, au début de la guerre du Golfe, le Préfet de Région convoque des personnalités religieuses, qui lancent un appel à la fraternité et au dialogue entre juifs, chrétiens et musulmans. À Aubervilliers, en octobre 2000, le maire et le proviseur organisent un débat avec des représentants religieux dans un lycée public. À Bobigny, le maire invite les chefs religieux à se rencontrer à la mairie après les attentats du 11 septembre, puis en avril 2002, suite à l'agression d'un jeune juif. Des municipalités organisent des conférences sur le fait religieux ou subventionnent une association interreligieuse. C'est le caractère plurireligieux qui rend ces initiatives ou ces soutiens publics possibles en contexte laïque.

8 À l'échelon national, des responsables politiques se font aussi promoteurs de l'entente interreligieuse. Le 20 septembre 2001, le président de l'Assemblée nationale (R. Forni) convoque quatre responsables religieux ${ }^{22}$ et déclare à la sortie que « dialoguer avec les religions, c'est instaurer une collaboration intelligente entre nous, permettant de combattre le terrorisme international ${ }^{23}$. En 2003, le ministre de l'Intérieur (Nicolas Sarkozy) développe en divers lieux sa pédagogie de l'entente interreligieuse. Il explique dans un colloque judéo-chrétien que «le paradis est le même » pour les trois religions : «Amis juifs, chrétiens, ou amis musulmans, dans la vie après la mort, si ce Dieu existe, ce sera le Dieu de tous ${ }^{24}$. Il interpelle les responsables religieux : «Le devoir des hommes de religion est de donner l'exemple de ce dialogue interreligieux dont nous avons tant besoin. Si vous hommes de foi, porteurs d'espérance, vous ne donnez pas l'exemple, comment les autres, ceux qui éprouvent la fragilité des choses et la rareté du temps, pourraient dialoguer » et insiste sur la nécessité de s'engager dans un dialogue avec les musulmans, sans lesquels « le dialogue interreligieux ne sera pas complet ». Le président de l'UDF (Union pour la Démocratie Française), François Bayrou affirme lors d'une soirée pour la paix en Irak organisée par une fédération musulmane : «Si nous prenons au sérieux ce que nous croyons, juifs, chrétiens, musulmans, nous croyons en un Dieu unique [...] il faut bien que nous confessions que c'est le même Dieu. Cela devrait mener à la fraternité. Quant à ceux qui ne croient pas en Dieu, ceux-là croient en l'homme " ${ }^{25}$. Des élus participent ou assistent à certaines célébrations ou conférences interreligieuses, organisées par des associations interreligieuses locales, ou plus rarement à celles qu'initient des groupes religieux ${ }^{26}$.

9 Au plan local comme national, les acteurs religieux sont conscients de l'avantage que les acteurs politiques retirent de ces juxtapositions plurireligieuses, comme l'affirme ce prêtre: "les maires voudraient bien une religion civile et pour eux ce serait l'interreligieux [...] c'est leur nouvelle manière de faire avec la persistance du religieux [...]. Il peut y avoir plusieurs religions, mais capables de cohabiter ». Le président de la Fédération Protestante souligne même l'ambiguïté du «politiquement correct de 
l'interreligieux» et observe que le succès de l'opération de Forni est attesté par le nombre de journalistes présents plus que par l'intérêt très limité que les présidents des groupes politiques portent aux exposés des responsables religieux ${ }^{27}$.

10 Ces manifestations plurireligieuses, locales ou nationales, montrent certes que la crainte d'une "guerre des dieux ${ }^{28}$ est présente chez des religieux comme chez des nonreligieux. Elles contribuent aussi à une certaine visibilité des religions sur la scène publique, qu'elles soient anciennement ou récemment établies, mais avec des choix variables concernant à la fois le degré d'élargissement de la pluralité et le type de représentants ou de courants conviés.

L'État laïque et les religions : quelle reconnaissance, quelle égalité ?

11 La prise en compte de la pluralité des groupes religieux par l'État et par les représentants des pouvoirs publics soulève alors trois types de questions: comment sont choisis les «bons » représentants? Quel est le type de reconnaissance accordée? Quel est le degré d'égalité entre les groupes?

Le choix des « bons » représentants

12 Les divers types de mises en scène publique de la pluralité religieuse contribuent, comme on vient de le voir, à une certaine accréditation des religions «minoritaires » ou nouvellement installées. Elles montrent aussi que, pour les acteurs politiques, travailler avec plusieurs groupes religieux permet de choisir les «bons» interlocuteurs, en particulier musulmans, tout en ayant l'appui de représentants d'autres religions et de laïcs qui approuvent cette prise en compte de la pluralité religieuse. Cette promotion des relations intercommunautaires va alors souvent de pair avec une conception extrêmement extensive de l'intégrisme, incluant toute religiosité visible, populaire ou «orthodoxe». La reconnaissance par l'autorité publique de groupes religieux s'accompagne donc d'une définition implicite du « religieusement acceptable » et a par conséquent pour effet secondaire la catégorisation des groupes non choisis comme interlocuteurs en groupes « intégristes » ou « dangereux », dont l'image auprès du public se trouve encore davantage détériorée.

13 On observe aussi que la reconnaissance par des pouvoirs publics locaux d'une organisation religieuse peut être facilitée par le fait que certains de ses responsables participent activement à des activités interreligieuses de type associatif ou religieux. Cette participation rassure et donne une sorte de caution concernant leur " ouverture ", comme le souligne le responsable d'une association musulmane locale, qui après plusieurs années de parcours d'obstacle (préemptions...), obtient la possibilité de louer un local municipal: «le fait qu'on soit dans l'interreligieux, on nous l'a dit, ça a joué, ça les a rassurés».

L'« interreligieux », un dispositif supplémentaire

de reconnaissance des cultes?

14 Il faut souligner que s'il n'existe pas de dispositif de reconnaissance des cultes en France, les groupes religieux peuvent faire des démarches qui leur assurent certains avantages, en particulier fiscaux. L'État accorde des exonérations fiscales à des institutions ou associations cultuelles auxquelles il octroie ou non la "grande capacité juridique », il rémunère des aumôniers hospitaliers, pénitentiaires et de l'armée, contribue à l'entretien d'édifices cultuels, subventionne des établissements d'enseignement privés ${ }^{29}$. Un autre type de reconnaissance est celui des créneaux spécifiques accordés à certains groupes religieux dans les médias (radio et télévision publiques). Un troisième type de 
reconnaissance est celui de la cérémonie des vœux au président de la République, qui n'a été élargi à la représentation musulmane qu'en janvier 2004, donc après la création du Conseil Français du Culte Musulman ${ }^{30}$.

Dans cette configuration, où l'État laïque ne reconnaît théoriquement aucun culte, mais où certains dispositifs légaux, médiatiques ou «symbolico-politiques " révèlent une reconnaissance différenciée des groupes religieux présents sur le territoire national, on peut alors se demander si l'«interreligieux» peut jouer le rôle d'un dispositif supplémentaire de reconnaissance implicite des religions. Pour répondre à cette question, il est nécessaire de distinguer les groupes dont le caractère religieux ne porte pas à discussion, ceux qu'on qualifie parfois de "grandes religions ", des groupes religieux très minoritaires et peu connus, qui font face au risque d'être qualifiés de sectes.

Le catholicisme, le protestantisme, l'orthodoxie (chrétienne), le judaïsme, l'islam et le bouddhisme sont considérés comme faisant partie des "grandes religions" ${ }^{31}$. L'« interreligieux» est pour ces groupes une occasion de reconnaissance de leur pertinence sociale et symbolique et une affirmation publique de la pluralité religieuse et de l'égalité des cultes. Il suffit de voir comment la participation de divers responsables religieux est médiatisée lors de cérémonies interreligieuses en temps de crise (Guerre du Golfe, 11 septembre 2001) ou d'actes de profanations de la mémoire (cimetière juif d'Herrlisheim en 2004).

Pour les groupes religieux très minoritaires, notamment les hindous, les néo-hindous comme les brahma kumaris, les adventistes ou les bahaïs, la reconnaissance accordée par un dispositif interreligieux s'avère bien plus déterminante que pour les autres groupes. En effet, la participation à de tels dispositifs leur accorde implicitement le statut de religion, reconnue par leurs "pairs ${ }^{32}$. Il n'est alors pas surprenant, que du côté des groupes religieux controversés, l'«interreligieux» soit aussi vu comme un moyen d'obtenir une reconnaissance et une respectabilité. Alors que des représentants de l'Église de Scientologie ont vainement tenté d'intégrer une organisation interreligieuse (la Conférence Mondiale des Religions pour la Paix), l'Église de l'Unification (Moon) est maintenant essentiellement active en France à travers les activités interreligieuses qu'elle organise, par le biais de son mouvement, la "Fédération interreligieuse et internationale pour la paix ».

On observe donc dans certains dispositifs interreligieux (associatifs, ou ponctuel en temps de crise) que les religions « reconnues » cooptent en quelque sorte des représentants de celles qui le sont moins (en choisissant le cas échéant les tendances qu'il est souhaitable de coopter) et contribuent de ce fait à leur degré d'accréditation sur la scène publique.

L'État peut-il promouvoir l'égalité religieuse?

On assiste à une autre transformation plus intéressante encore et tout à fait nouvelle : l'État et les pouvoirs publics deviennent directement acteurs de l'évolution de la laïcité en commençant à prendre en compte l'écart entre l'égalité formelle et l'égalité réelle, ou entre liberté formelle et liberté réelle, dans le domaine religieux. Dans le domaine religieux, la formulation de l'égalité formelle apparaît implicitement dans les principes de liberté religieuse, d'interdiction de discrimination fondée sur la religion, dans l'affirmation que la République « respecte toutes les croyances ${ }^{33}$.

20 Le défi de la réduction de l'écart entre l'égalité formelle et l'égalité réelle n'est pas neuf, du moins dans les domaines économique et éducatif. Pour des raisons à la fois idéales (de justice) et pragmatiques (de paix sociale), l'État a déjà reconnu qu'il ne peut pas être 
complètement neutre. Il a donc instauré diverses mesures visant à assurer des revenus minimaux et un accès à la santé (droits économiques et sociaux) ou à faciliter les projets éducatifs (zones d'éducation prioritaire). On peut d'ailleurs souligner, suivant en cela l'analyse de Dominique Schnapper, que les politiques de la Ville sont de fait une forme d'ethnicisation de l'action de l'État-providence ${ }^{34}$, puisqu'elles visent les «quartiers sensibles ", qui sont le plus souvent des quartiers "ethniques" (et à forte présence musulmane).

De manière parallèle, même si c'est de façon moins marquée, l'État semble aujourd'hui reconnaître qu'il ne peut plus rester complètement neutre vis-à-vis de la pluralité religieuse et des diverses difficultés auxquelles font face les groupes religieux « minoritaires ». Il intervient de plus en plus fréquemment pour promouvoir une certaine protection de la différence, un certain "droit à l'identité religieuse », en reconnaissant les religions « minoritaires » et en favorisant une certaine connaissance mutuelle.

La reconnaissance de ce "droit» entre évidemment en tension avec l'ambition de transcender par la citoyenneté les appartenances ou les fidélités particulières. Les obstacles et les résistances à cette égalité sont massifs et la réalité est souvent une inégalité flagrante. Ainsi, à propos des prisons, Farhad Khosrokhavar souligne qu'« on observe une inégalité incontestable dans la manière dont l'islam est pris en charge par l'institution carcérale, en comparaison notamment avec le catholicisme, le protestantisme et le judaïsme ${ }^{35}$. L'univers carcéral (comme l'armée et l'hôpital) est pourtant un lieu où la législation laïque prévoit que l'État doit subvenir aux besoins spirituels des personnes privées de liberté. La difficulté de la mise en œuvre de l'égalité est aussi apparue de manière criante dans le traitement de la question des signes religieux dans l'espace public, par exemple dans le fait qu'en 2003, la commission Stasi sur la laïcité n'ait auditionné que deux femmes portant le foulard et seulement lors de sa dernière séance ${ }^{36}$. On pourrait multiplier les exemples d'inégalités réelles, en observant les difficultés liées à l'implantation de lieux de culte. Une fois soulignée la persistance de ces inégalités, on doit cependant constater que, même dans un État républicain où la reconnaissance du groupe semble contradictoire ou pour le moins problématique, la reconnaissance de l'autre, au niveau de l'individu comme du groupe, apparaît de plus en plus comme une exigence démocratique incontournable.

La question est alors de savoir comment promouvoir l'égalité réelle, au-delà des déclarations de principe. Cette promotion passe pour l'instant surtout par la mise en situation d'égalité (symbolique du moins) des religions «minoritaires » avec la religion majoritaire. On observe depuis une quinzaine d'années que les lieux d'affirmation de cette égalité symbolique se multiplient, à l'échelle locale ou nationale. Il peut s'agir de cérémonies de deuil ou de prières pour la paix, organisées de manière plurireligieuse, qui se sont développées depuis la Guerre du Golfe, comme après l'assassinat des moines de Tibhirine (1996), le crash du Concorde (2000), le 11 septembre (2001) ${ }^{37}$, ou encore des diverses formes de promotion de la pluralité religieuse coopérative qui ont été mentionnées dans la première partie (déclarations communes, réception de responsables religieux par des acteurs politiques, participation à des comités consultatifs, diffusion de la connaissance de la diversité religieuse par des conférences, des rencontres ou des actions éducatives). L'État et les pouvoirs publics accompagnent leur toute relative et embryonnaire reconnaissance de la diversité par une promotion de la rencontre des religions et une affirmation du dépassement possible des antagonismes ${ }^{38}$. Ces interventions sont évidemment loin d'être structurées et systématisées, mais elles se 
multiplient à l'échelle locale et nationale. Dans cette dynamique, certains groupes ou partenaires sont cependant plus égaux que d'autres. En effet, comme on l'a vu plus haut, la reconnaissance publique de la pluralité religieuse va de pair avec le choix des «bons » partenaires au sein d'un groupe religieux. L'égalité réelle concernera donc surtout les groupes religieux et les tendances au sein de ces groupes ou les représentants qui sont à la fois reconnus par les pouvoirs publics et par les interlocuteurs religieux déjà reconnus.

Si la promotion de l'égalité réelle demeure pour l'instant le plus souvent déclarative, sa mise en scène publique lui fait cependant dépasser son caractère purement programmatique ou idéal. Elle contribue à renforcer le sentiment d'inacceptabilité des écarts constatés sur le terrain, aussi bien pour les groupes discriminés (qui y voit une légitimation à leurs revendications d'égalité) que pour le grand public. Ces actions, quelle que soit leur nature, sont bien une forme (ou un début) de "politique de reconnaissance ", selon l'expression du philosophe canadien Charles Taylor, qui montre que celle-ci ne s'oppose pas à la "politique d'égale dignité ", et peut même en être une conséquence, si l'égalité concerne aussi la possibilité d'avoir une identité propre ${ }^{39}$.

Ces changements sont sociaux et politiques, dans les formes concrètes qu'ils prennent et dans la rhétorique qui les accompagne. Ils correspondent aussi à un changement à la fois philosophique et mettant en jeu la vision anthropologique ou l'ontologie sociale, et nécessitant de dépasser l'antagonisme entre deux conceptions de la relation entre individus et groupes sociaux. La première conception correspond à la vision individualiste libérale, qui néglige le rôle des groupes et semble avoir la faveur du régime républicain français ${ }^{40}$. La seconde conception, souvent qualifiée de communautarienne, accorde une plus grande importance au rôle des groupes et aux appartenances et peut être défendue, dans une version plus ou moins accentuée et de manière réactive, par des groupes insuffisamment reconnus. Face à ces deux conceptions, il serait certainement plus pertinent et plus opératoire de reconnaître d'une part que l'État et ses porte-parole (hommes politiques ou experts) sont aussi des acteurs ethniques ${ }^{41}$, et d'autre part de mettre en œuvre une conception relationnelle du rapport de l'individu aux groupes sociaux. Dans cette approche relationnelle, les individus sont porteurs d'identités composites et dynamiques. Ces identités se construisent par des appartenances, ellesmêmes multiples, des relations à des autres, individus et groupes, et des orientations vers le bien moral, des valeurs ${ }^{42}$. Cette conception prend en compte la dimension culturelle et symbolique des rapports sociaux, mais ne néglige aucunement leurs dimensions politique et économique. Les acteurs des relations interreligieuses ou interethniques sont membres de groupes sociaux et les rapports de pouvoir économiques, politiques et symboliques existent à la fois au sein de ces groupes et entre ces groupes.

Les groupes religieux ont à l'évidence besoin de la reconnaissance de l'État, mais il apparaît aussi que l'État laïque reconnaît une certaine utilité aux religions, comme ressources d'éthique, de sens et de lien social, d'autant plus que, comme le souligne Marcel Gauchet, le « désenchantement » du politique confère un rôle accru à la société civile : cette «mutation fondamentale de la politique démocratique tend à réinsuffler [aux identités religieuses] une dignité et une utilité nouvelles, en fonction des besoins même de la sphère publique, en tant que systèmes généraux de sens, ou doctrine globale des fins ${ }^{43}$. Au-delà du rôle d'intégration et de travail social que joue chacun des groupes religieux, la « pluralité active » apparaît comme réductrice d'antagonismes et promotrice d'une certaine reconnaissance. Deux facteurs favorisent cette reconnaissance: d'une part, la proximité, dans des contextes marqués par un multiculturalisme de fait et donc 
par la pluralité religieuse et d'autre part, la mise en scène de la pluralité : lors de grands colloques, de cérémonies interreligieuses, qui rassemblent jusqu'à plusieurs milliers de participants ou encore lors des invitations plurireligieuses officielles, à l'initiative de pouvoirs publics locaux ou nationaux. Ces dernières situations contribuent à une reconnaissance « de fait » de la diversité religieuse.

Sous l'effet de la pluralité, la laïcité est en train d'évoluer vers une plus grande prise en compte de la diversité des identités culturelles, ethniques et religieuses en France. L'« interreligieux » en général, et plus particulièrement dans sa dimension " politique » que nous venons de traiter, contribue à la fois à redonner une certaine place aux religions dans l'espace public et à la définition du « religieusement correct ». Ces dynamiques vont de pair avec une dimension gestionnaire de la part de l'État et des pouvoirs publics, qui instrumentalisent cette promotion de la pluralité pour reconnaître certains groupes, tendances ou représentants plutôt que d'autres. Dans ces processus de reconnaissance, de différenciation ou de choix de partenaires, les représentants de l'état ne sont évidemment pas des acteurs neutres. Ils sont aussi des acteurs « ethniques", porteurs de la culture majoritaire, face aux "nouveaux entrants ", aspirant à la pleine citoyenneté, sans pour autant renoncer aux spécificités de leur identité et de leur mémoire.

\section{NOTES}

1. Pierre Bréchon, Bruno Duriez, Jacques Ion, dirs., Religion et action dans l'espace public, Paris, L'Harmattan, 2000 ; Gilbert Vincent, dir., La place des ceuvres et des acteurs religieux dans les dispositifs de protection sociale, Paris, L'Harmattan, 1997 (cf. Arch. 118.74).

2. Frank Frégosi et Jean-Paul Willaime, éds., Le religieux dans la commune. Régulations locales du pluralisme en France, Genève, Labor et Fides, 2001 (coll. « Histoire et Société ») (cf. Arch. 124.20).

3. Anne-Sophie Lamine, La cohabitation des dieux. Pluralité religieuse et laïcité, Paris, PUF, 2004.

4. Cet article n'aborde donc pas les relations entre l'État ou les pouvoirs publics locaux et un groupe religieux particulier.

5. Ces termes « majoritaire » et « minoritaire » peuvent présenter l'inconvénient d'une référence implicite au « majoritaire » comme référence. De plus que s'il s'agit souvent d'un rapport entre le groupe le plus important (catholique) et tous les autres, dans certains cas, le rapport se joue plutôt entre les religions anciennement établies et celles récemment implantées sur le territoire (dans ce cas le premier groupe inclut aussi les protestants et les juifs, alors que les musulmans, bien plus nombreux sont dans le second groupe).

6. Pour une présentation plus détaillée, voir Anne-Sophie Lamine, « Quand les villes font appel aux religions », Annales de la recherche urbaine, $\mathrm{n}^{\circ}$ 96, 2004, p. 149-156.

7. L'agglomération millionnaire compte environ 500000 catholiques, 180000 musulmans, 75000 juifs, 20000 protestants, 10000 orthodoxes, 5000 bouddhistes et 80000

Arméniens. 
8. Dont le choix est tacitement soumis à l'approbation de l'adjoint.

9. Début de la guerre du Golfe (1991), meurtre d'un jeune rappeur d'origine comorienne par des colleurs d'affiches du Front National (1995), meurtre d'un jeune homme par un adolescent d'origine maghrébine (1996), assassinat des moines de Tibhirine (1996), début de la seconde Intifada (2000), attentats de New-York (2001), incendie d'une synagogue (2002), guerre d'Irak (2003).

10. L'Institut Paoli-Calmettes de traitement des cancers.

11. Philippe Aziz, Le Paradoxe de Roubaix, Paris, Plon, 1996. Ces thèses sont reprises par l'hebdomadaire Le Point. Le maire dénonce alors la « désinformation » et affirme que la population d'origine étrangère compte pour environ $30 \%$.

12. Suite à des changements de responsables, le nombre de participants musulmans a ensuite baissé. La pression de la mairie pour le passage en associations régies par la loi de 1905 semble y avoir aussi contribué.

13. La ville ne compte que quelques familles juives et pas d'organisation, mais « une place leur est réservée ».

14. Les fêtes proposées sont chrétiennes (Noël, Épiphanie, Chandeleur, Pâques), musulmane (Aïd el Khébir), bouddhiste (Vesak), chinoise (Nouvel an chinois) et laïques (Fête de la rentrée, $1^{\text {er }}$ mai, Fête de l'amitié).

15. Donc régies par la loi de 1905 : mise à disposition d'un terrain municipal par bail emphytéotique, location de bâtiment et subvention de réparations d'édifices affectés au culte.

16. La ville entend « apporter sa contribution à une meilleure connaissance des diverses croyances sur un plan strictement laïque, [...faire] reculer l'ignorance, contribuera à l'établissement d'un climat de dialogue et de respect. Le projet de construction d'une mosquée est également issu de ce processus. » (Conseil Municipal du 02/05/02 ; on vote aussi une subvention pour la réparation de l'église du centre ville).

17. Les participants sont retraités (50\%), agents de diverses institutions (de Montreuil et d'autres villes), mais peu d'enseignants en activité (les cours ont lieu le mercredi aprèsmidi).

18. En 1997, dans le journal municipal, le maire met en garde contre du soutien scolaire " préalable à des cours coraniques [...] où on inculque aux enfants une idéologie en contradiction manifeste avec les principes fondamentaux de l'éducation laïque et républicaine ». L'association existe alors depuis deux ans et propose des cours d'arabe, d'éducation religieuse et une salle de prière (Islam de France, 2, 1998, p. 160-164).

19. Une chargée de mission de la mairie commente : « les juifs ont embrassé les musulmans ».

20. Églises catholique, orthodoxe, réformée, anglicane, luthérienne, baptiste, arménienne apostolique, judaïsme et islam. La mairie de Cergy-Pontoise a mis en place en 2003 une "Commission des religions", comprenant quatre membres de chacune des confessions catholique, protestante, juive et musulmane et dont une des premières actions a été d'organiser une " cérémonie interreligieuse pour la paix », le 11 novembre 2003.

21. Selon les termes des communiqués de la mairie de Paris.

22. Le cardinal Louis-Marie Billé, le recteur Dalil Boubakeur, le grand rabbin Joseph Sitruk et le pasteur Jean-Arnold de Clermont.

23. Bulletin d'Information Protestant $n^{\circ} 1525$, octobre 2001, où on lit ce commentaire « le succès de la cérémonie interreligieuse à l'Église américaine à Paris, n'ayant échappé à personne [R. Forni] a souhaité associer à l'image de l'Assemblée nationale, l'entente bienvenue entre les responsables religieux en France ». 
24. Le 10 mars 2003, "Secondes Rencontres européennes entre juifs et catholiques ", Mairie de Paris, 1200 participants. Il reprend la même argumentation peu après lors de l'assemblée générale de la Fédération Nationale des Musulmans de France et développe aussi cet argumentaire dans l'interview réalisée par Henri Tincq « La laïcité n'est pas l'adversaire de la religion ", Le Monde des Religions, $\mathrm{n}^{\circ}$ 1, septembre 2003, p. 66-69. 25. «Soirée pour la paix en Irak », organisée aussi par l'Union des Associations Musulmanes de Seine-Saint-Denis, en présence de personnalités politiques et religieuses à Drancy, le 28 janvier 2003.

26. L'interreligieux organisé par un groupe religieux étant souvent de nature plus religieuse (dans les thèmes abordés), la présence d'élus y est plus rare, sauf dans le cas où il est organisé par un groupe « minoritaire » qui recherche cette dimension de reconnaissance publique et les invite (par exemple lors d'une célébration de l'Aïd où des représentants de diverses religions et des élus sont invités).

27. Colloque « Le dialogue interreligieux : chance ou défi », Fédération Protestante, Paris, 28 septembre 2002.

28. Max Weber, Le savant et le politique, Paris, Plon, 1997 ; Raymond Boudon, Le sens des valeurs, Paris, PUF, 1999 ; Sylvie Mesure et Alain Renaut, La guerre des dieux. Essai sur la querelle des valeurs, Paris, Grasset, 1996 ; Samuel P. Huntington, Le choc des civilisations, Paris, Odile Jacob, 1997 ; et la fine critique de Marc Crépon, L'imposture du choc des civilisations, Paris, Pleins Feux, 2002.

29. Les déductions sur les dons effectués aux associations cultuelles et les possibilités de recevoir des legs, au titre de la loi de 1905 ne sont pas automatiquement accordées aux associations déclarées cultuelles (voir le rapport de Brigitte Gaudemet-Badesvant, 1998, sur la liberté confessionnelle et le régime juridique des cultes www.conseilconstitutionnel.fr/divers/internat/ libreg.rtf).

30. Les cultes participant à la cérémonie de vœux sont le catholicisme, le protestantisme et le judaïsme.

31. Dans certains cas (représentation publique, mémoire), le bouddhisme et l'orthodoxie ne sont pas conviés, mais leur statut de religion n'est pas mis en cause.

32. Cette reconnaissance est très variable, selon les groupes et leur implantation locale. Il est cependant intéressant de noter qu'une des questions posées par un membre du ministère de la Jeunesse et des Sports à un responsable bahaï au moment où il faisait des démarches fut : «Est-ce que les autres religions vous reconnaissent comme une religion et vous invitent aux réunions interreligieuses?»

33. En particulier dans l'article 2 de la Constitution Française.

34. Dominique Schnapper, La démocratie providentielle, Paris, NRF Essais, Gallimard, 2002, p. $175-218$.

35. Farhad Khosrokhavar, L'islam dans les prisons, Paris, Balland, 2004, p. 13.

36. Anne-Sophie Lamine, « Die Republik, die Schule und die Kopftücher », Religion-StaatGesellschaft, 2/2004 (« La République, l'école et les foulards »).

37. Sous la forme d'une juxtaposition de prières de diverses traditions religieuses (le plus souvent juive, catholique, protestante et musulmane, parfois avec une participation plus large, à l'échelle locale), pouvant aussi inclure une prise de parole de responsables communautaires nationaux ou locaux. La participation de représentants politiques leur donne une dimension " officielle ".

38. En plus des cas déjà évoqués, on peut citer la déclaration du ministre de l'Intérieur (Dominique de Villepin), en juin 2004, après la profanation de tombes musulmanes en Alsace (dans un contexte suivant la profanation de tombes juives), qui souhaite « la 
création d'un conseil des cultes dans les départements ", comprenant des représentants des différentes religions et en lien avec les élus.

39. Charles Taylor, Multiculturalisme, Paris, Flammarion, 1994.

40. On peut cependant souligner comme le fait Philippe Raynaud que certains « théoriciens du républicanisme à la française », bien qu'ils se posent en adversaires du communautarisme, sont philosophiquement communautaristes (et antimulticulturalistes) «Diversité de la diversité », Quaderni, 47, 2002, p. 47-55.

41. La culture « majoritaire » est bien aussi une culture particulière et ceux qui s'y réfèrent implicitement ou explicitement interviennent bien dans la construction sociale de la différence. Albert Bastenier, Qu'est-ce qu'une société ethnique. Ethnicité et racisme dans les sociétés européennes d'immigration, Paris, PUF, 2004, p. 193. L'identité religieuse est un mode important de la déclinaison de la différence ethnique, mais non le seul.

42. Sur la construction de l'identité, voir Charles Taylor, Les sources du moi. La formation de l'identité moderne, Paris, Seuil, 1998. Pour une discussion des divers types d'ontologies sociales, voir David Kahane, « Délibération démocratique et ontologie sociale », Philosophiques, 29/2, 2002, p. 251-286.

43. Marcel Gauchet, La religion dans la démocratie. Parcours de laïcité, Paris, Folio-Essai, 2001, p. 135.

\section{RÉSUMÉS}

Cet article examine la contribution des pratiques de l'« interreligieux » à la reconnaissance des religions dans l'État laïque. Il montre qu'à côté du mouvement associatif interreligieux et des activités organisées par les religions elles-mêmes se développent des pratiques politiques de promotion de la «bonne entente des religions ». Il analyse leurs enjeux en termes de choix des représentants et d'égalité entre les groupes religieux.

This article shows the contribution of interfaith activities towards the recognition of religion by the secular State. Besides interfaith organisations and interreligious activities carried out by religious groups, political practices of promoting "harmony between religous groups" are developped. It analyses their stakes in terms of equality and choice of sing representatives.

Este artículo examina la contribución de las prácticas "interreligiosas" al reconocimiento de las religiones en el Estado laico. Muestra que al lado del movimiento asociativo interreligioso y de las actividades organizadas por las mismas religiones, se desarrollan prácticas políticas de promoción del "buen entendimiento entre las religiones". Analiza sus apuestas en términos de elección de los representantes y de igualdad entre los grupos religiosos. 


\section{AUTEUR}

\section{ANNE-SOPHIE LAMINE}

Université Marc Bloch

Laboratoire Cultures et Sociétés en Europe, Strasbourg 